\title{
Flexible Fibreoptic Bronchoscopy Performed Through a Laryngeal Mask Airway (FFB-LMA) in a Paediatric Population: Experience of a Tertiary Care Children's Hospital in Sri Lanka
}

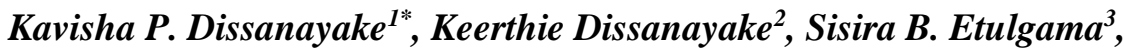 \\ Kapila Hettiarachchi ${ }^{4}$ \\ ${ }^{1}$ Senior Registrar in Anaesthesiology, ${ }^{3}$ Consultant ENT Surgeon, ${ }^{4}$ Consultant Anaesthetist, Sirimavo \\ Bandaranayake Specialized Children's Hospital, Peradeniya, Sri Lanka, ${ }^{2}$ Doctoral Student, Institute of \\ Biomedicine and Translational Medicine, University of Tartu, Tartu, Estonia
}

\begin{abstract}
Studies suggest that flexible fibreoptic bronchoscopy, when performed through a laryngeal mask airway (FFB-LMA), have fewer rates of complications and better efficacy. In comparison to rigid bronchoscopy, FFB is safer for the operator, as it prevents direct exposure to respiratory tract infections such as Covid-19. Airway maintenance with LMA is uncommon when performing FFB in Sri Lanka. This study aimed to report the efficacy and peri-procedural complications of FFB performed through LMA at a tertiary care children's hospital in Sri Lanka. Data were retrieved retrospectively from anaesthetic charts and bed head tickets of all the patients who underwent FFBs performed through LMA at the Sirimavo Bandaranayake Specialized Children's Hospital, Peradeniya. Collected data included age, gender, indication, duration of the procedure, bronchoscopy findings, and complications, if any. Of the total of 176 patients, the majority were infants and between $1-5$ years $(33.52 \%$ each). The most common indication was recurrent/persistent respiratory tract infections $(57.9 \%)$ followed by foreign body aspirations (21.6\%). Clinically significant abnormalities were detected in $87 \%$ of the cases. The success rate of extraction of positively identified foreign bodies was $100 \%$. Perioperative complications were observed in $4.5 \%$ of the patients. Risk factor analysis, based on multivariate logistic regression, identified the duration of the procedure as a risk factor for complications $(\mathrm{OR}=15.57, \mathrm{p}=0.0011)$. FFB performed through LMA has good efficacy and low complication profile in the studied patient sample.
\end{abstract}

Key words: flexible fibreoptic bronchoscopy, laryngeal mask airway, paediatric anaesthesia, ENT surgery

\section{Introduction}

Paediatric flexible bronchoscopy is a routine procedure carried out to diagnose different pulmonary pathologies and therapeutic interventions. ${ }^{1}$

*Correspondence: Kavisha P. Dissanayake

E mail: kavisha.premasinghe@ gmail.com

https://orcid.org/0000-0002-2987-3185

Received: $10 / 10 / 2020$

Accepted: 28/01/2021

DOI: https://doi.org/10.4038/slja.v29i2.8693

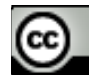

Since its introduction in the 1970s, it gradually replaced the rigid bronchoscopy. Over the years, the technology and safety profile of flexible bronchoscopy has improved.

Diagnostic delay or failure to extract aspirated foreign bodies from airways have led to significant morbidity and mortality, especially in developing countries. $^{2}$ For the extraction of asphyxiating foreign bodies, rigid bronchoscopy is considered the mainstay of intervention, compared to flexible bronchoscopy. ${ }^{3}$ However, still, there are many complications and limitations in the use of rigid bronchoscopy, including higher rates of desaturation, longer procedural duration, and limited access to distal airways. ${ }^{4}$ Most of these 
could be overcome by performing FFB through a laryngeal mask airway (FFB-LMA). Having a larger internal diameter, compared to endotracheal tubes, LMA allows the passing of larger diameter flexible bronchoscopes and associated ancillary tools. ${ }^{5}$ Moreover, convenience to share the airway, 210-degree view, lower shunt fraction thus longer maintenance of oxygen saturation, lower incidence of physical trauma and temporomandibular joint injuries and no necessity of complete muscle relaxation are advantages of FFB compared to rigid bronchoscopy. Many hospital-based studies have evaluated the effectiveness and experience with FFB-LMA in children. ${ }^{6,7}$ To the best of our knowledge, the experience of using FFB-LMA in a Sri Lankan paediatric patient cohort has not been reported. Such knowledge is vital to understand the efficacy and safety of FFB-LMA in paediatric patient care for improving its current standards.

In this study, we aimed to retrospectively analyse the efficacy and complications of FFB-LMA in paediatric patients at a specialized paediatric hospital in Sri Lanka.

\section{Methodology}

The study was approved by the institutional ethical review committee, Faculty of Medicine, University of Peradeniya, Sri Lanka. Data were collected retrospectively from medical records including the anaesthetic monitoring charts, of patients who underwent FFB-LMA, at the Sirimavo Bandaranayake Specialized Children's Hospital, Peradeniya which is one of the first centres to perform this procedure in Sri Lanka. All the patients aged less than 12 years who underwent FFB-LMA between January 2014 - April 2017 were included. The standard procedure used when performing FFB-LMA was as follows. Induction was done with inhalation anaesthesia with sevoflurane or intravenous induction with propofol $(2 \mathrm{mg} / \mathrm{kg})$. Fentanyl $(1-2 \mu \mathrm{g} / \mathrm{kg})$ was given to all the patients. Anaesthesia was maintained with sevoflurane and boluses of propofol if needed. Total intravenous anaesthesia (TIVA) with propofol infusion was avoided to prevent adverse effects of propofol infusion such as bradycardia and hypotension. Intravenous suxamethonium $(1 \mathrm{mg} / \mathrm{kg})$ was used at the time of insertion of the bronchoscope to prevent patient movements, laryngospasm and bronchospasm. After inserting the LMA, a dual-axis swivel adapter was connected between the LMA and the breathing circuit, through which the bronchoscope was introduced into the patient's airway. A cut finger of a surgical glove was used to cover the port of entry

Figure 1.

Dual-axis swivel adapter was used for connecting the breathing circuit and LMA, and the insertion of the bronchoscope. Air leak was prevented by covering the port of entry of the bronchoscope to the swivel adapter using a cut finger of a surgical glove. A. Dual-axis swivel adapter illustrating the method used to prevent air leakage, $\boldsymbol{B}$. A bronchoscope passed through the swivel adapter and LMA, C. Performing FFB-LMA.

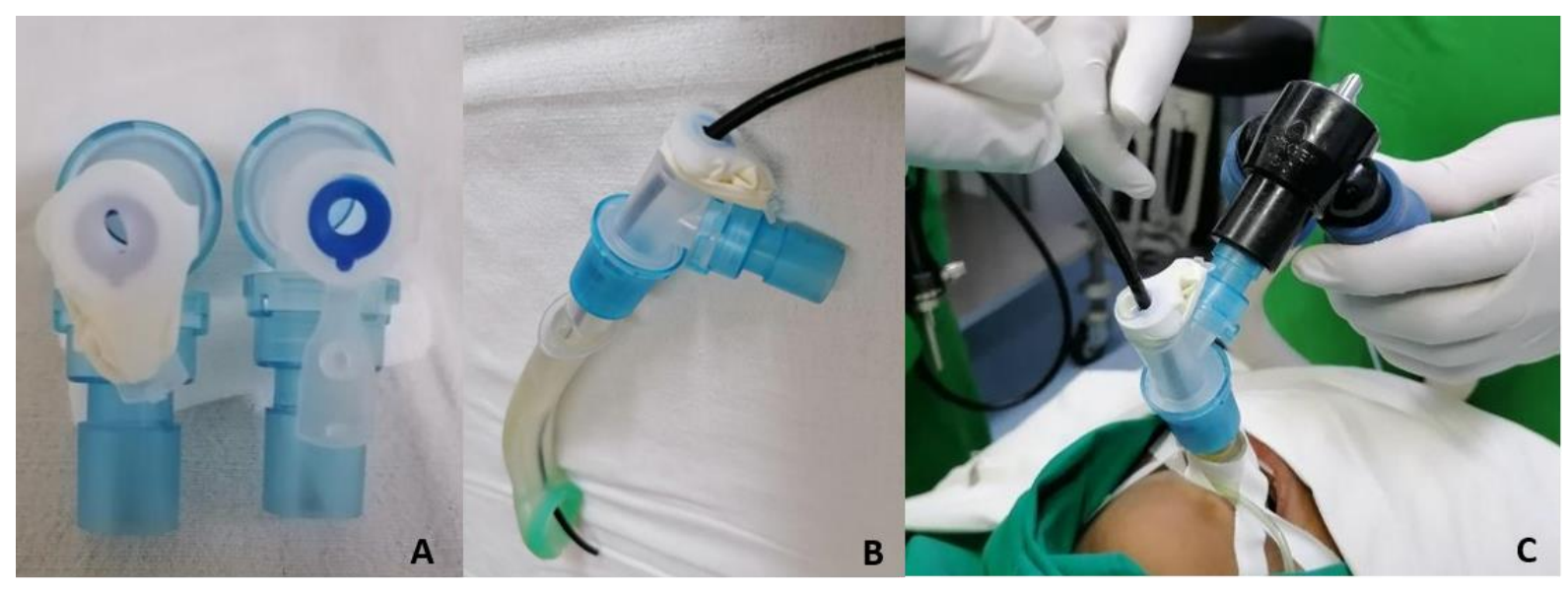


of the bronchoscope to the swivel adapter to prevent air leak during the bronchoscopy and ventilation (Figure 1). Manual ventilation was carried out to obtain better control and rapid adjustments of the depth of anaesthesia. Most of the FFB-LMA procedures were performed by an ENT surgeon while a few cases were done by trainees. Following bronchoscopy, LMA was safely removed once spontaneous breathing resumed with sufficient tidal volume and the patient was extubated in deep anaesthesia.

Patients were further monitored with heart rate, blood pressure, peripheral oxygen saturation $\left(\mathrm{SpO}_{2}\right)$, and end-tidal carbon dioxide $\left(\mathrm{EtCO}_{2}\right)$, and for complications at the recovery area for at least 30 minutes, before sending them back to the ward.

Data related to patient demographics, ASA grade (American Society of Anaesthesiologists physical status classification system), indications, abnormal findings and interventions, duration of the procedure, peri-procedural complications (both intra- and post-procedural) were recorded. Data regarding the duration of the procedure was obtained from the anaesthetic charts. Any deviation from the normal airway was considered as a clinically significant abnormality irrespective of the indication for the procedure. Among the recorded complications, desaturation was classified as minor desaturation (a drop of $\mathrm{SpO}_{2}$ up to $90 \%$ ) and major desaturation (a drop of the $\mathrm{SpO}_{2}$ below $90 \%){ }^{8}$

\section{Statistical analysis}

The efficacy/diagnostic yield, which is the percentage of cases identified as having a clinically significant abnormality out of all the FFB-LMA procedures were analysed considering patients of all age categories together. Risk factors for periprocedural complications were assessed using logistic regression analysis. After the univariate

Table 1: Characteristics of the patients and FFB-LMA

\begin{tabular}{|c|c|c|c|c|c|}
\hline & & $\begin{array}{l}\text { Neonates }(< \\
28 \text { days })\end{array}$ & $\begin{array}{c}\text { Infants } \\
(\geq 28 \text { days } \\
\text { and } \leq 1 \text { year })\end{array}$ & $\begin{array}{l}\text { Aged 1-5 } \\
\text { years }\end{array}$ & $\begin{array}{l}\text { Aged over } \\
5 \text { years }\end{array}$ \\
\hline \multicolumn{2}{|l|}{$\overline{\mathrm{N}}$} & 9 & 59 & 59 & 49 \\
\hline \multicolumn{2}{|c|}{ Gender ratio - male:female } & $1: 1$ & $2: 1$ & $7: 5$ & $1: 1$ \\
\hline \multirow{11}{*}{ Indication } & $\begin{array}{l}\text { Persistent/recurrent pneumonia } \pm \\
\text { consolidation } \mathrm{n}(\%)^{*}\end{array}$ & $2(22.22)$ & $42(71.18)$ & $28(47.45)$ & $31(63.26)$ \\
\hline & $\begin{array}{l}\text { Suspected foreign body aspiration } \\
\mathrm{n}(\%)\end{array}$ & - & $5(8.47)$ & $25(42.37)$ & $7(14.29)$ \\
\hline & Lung collapse n (\%) & - & $3(5.08)$ & $1(1.69)$ & - \\
\hline & H-type TOF-post operative, n (\%) & $5(55.56)$ & $1(1.69)$ & - & - \\
\hline & Stridor, n (\%) & $2(22.22)$ & $5(8.47)$ & - & $2(4.08)$ \\
\hline & Bronchiectasis, n (\%) & - & - & $2(3.38)$ & $2(4.08)$ \\
\hline & Haemangioma, n (\%) & - & - & - & $2(4.08)$ \\
\hline & Haemoptysis, n (\%) & - & - & - & $1(2.04)$ \\
\hline & Chronic cough, n (\%) & - & $1(1.69)$ & - & $1(2.04)$ \\
\hline & Persistent wheezing, n (\%) & - & $1(1.69)$ & - & $1(2.04)$ \\
\hline & Other* n (\%) & - & $1(1.69)$ & $3(5.08)$ & $2(18.36)$ \\
\hline \multicolumn{2}{|c|}{ Duration of bronchoscopy-median (IQR) } & $15(15-30)$ & $20(15-30)$ & $20(15-30)$ & $30(15-30)$ \\
\hline \multicolumn{2}{|c|}{ Intra-operative complications, n (\%) } & $1(11.11)$ & $4(6.77)$ & $3(5.08)$ & $2(4.08)$ \\
\hline \multicolumn{2}{|c|}{ Post-operative complications, n (\%) } & - & - & $1(1.69)$ & $1(2.04)$ \\
\hline \multicolumn{6}{|c|}{$\begin{array}{l}\text { FFB-LMA- flexible fiberoptic bronchoscopy performed through laryngeal mask airway, TOF - } \\
\text { tracheoesophageal fistula, IQR- inter-quartile ratio, n-number of patients, * percentages given within parenthesis } \\
\text { are the proportion of the patients within a given age category. }\end{array}$} \\
\hline
\end{tabular}


analysis, all the considered variables were included in the multivariate regression analysis. For data analysis and visualization MedCalc software (version 19.4) was used. $\mathrm{P}<0.05$ was treated as statistically significant. (suspected foreign body aspiration), $75 \%$ (persistent lung collapse), 66.66\% (suspected TOF) and $100 \%$ (stridor). Among the patients with abnormal findings for pneumonia, secretions and/or mucus plugs were removed in $70.5 \%$ and

Figure 2.

Outcome of FFB-LMA and interventions for all age categories. Data are presented under different indications. Percentages given within the parenthesis are the percentage of abnormal findings or interventions made for the given indication category. FFB-LMA- flexible fibreoptic bronchoscopy performed through laryngeal mask airway, FB-foreign body, TOF- tracheoesophageal fistula.

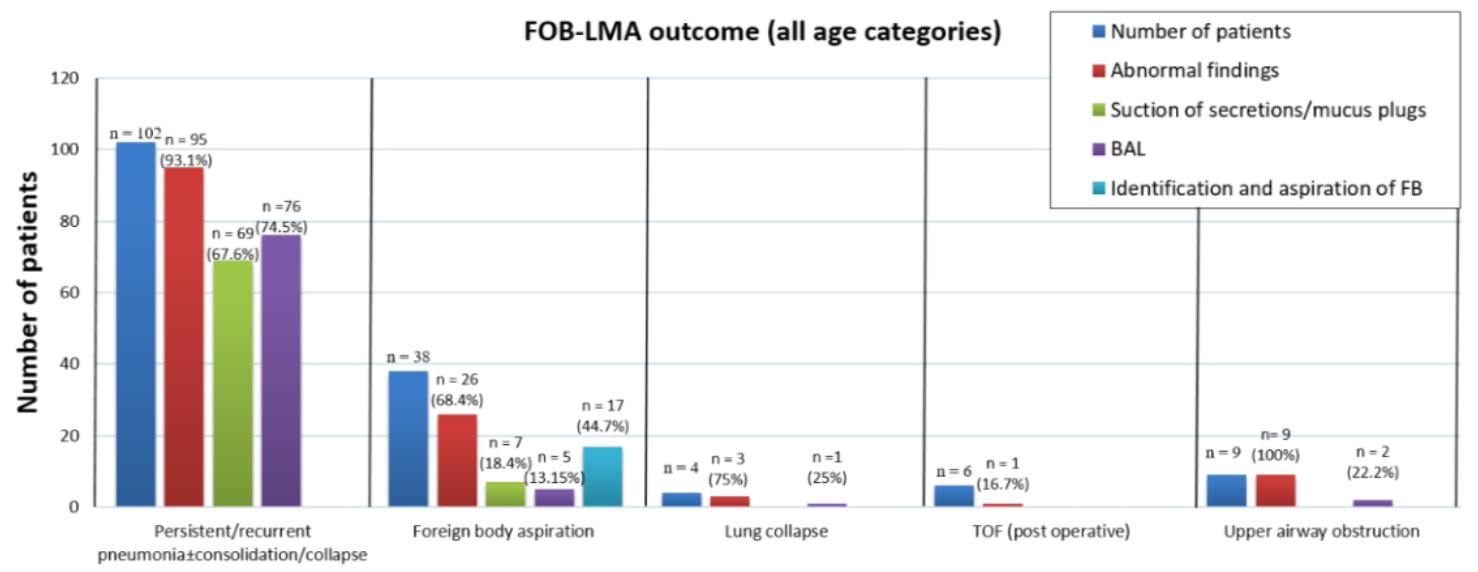

\section{Results}

Altogether 176 patients had undergone FFB through LMA. Of these, the majority $(\mathrm{n}=102$, $58 \%$ ) were males (gender ratio of 4:3). However, the gender ratio varied based on the age category (Table 1). Most patients were infants (33.52\%) and between $1-5$ years $(33.52 \%)$.

The most common indication for FFB was persistent/recurrent lower respiratory tract infection \pm consolidation $(\mathrm{n}=103,58.5 \%)$ followed by suspected foreign body aspiration ( $\mathrm{n}$ $=37,21 \%)$, stridor $(n=9,5.1 \%)$, post-operative $H$ type tracheoesophageal fistula (TOF) $(\mathrm{n}=6$, $3.4 \%)$, lung collapse $(\mathrm{n}=4,2.2 \%)$ and bronchiectasis $(\mathrm{n}=4,2.2 \%)$. Overall, clinically significant abnormalities were observed in $84.65 \%$ of cases. According to the main indications, the percent abnormal findings were $93.13 \%$ (persistent /recurrent pneumonia \pm consolidation), $68.42 \%$ bronchoalveolar lavage (BAL) was carried out in $80 \%$ of the patients (Figure 2). Among the patients who underwent the FFB-LMA to exclude TOF (post-operative or otherwise), though abnormal findings in the airway were noted in $66.6 \%$ of cases, none was positive for TOF. With regards to suspected foreign body aspiration, of the 38 patients investigated, 26 patients $(68.42 \%)$ had abnormal findings, and of them, only in 17 cases $(44.73 \%)$, foreign bodies were detected. However, in all 17 cases, the extraction of foreign bodies was successful. Only 1 case of congenital malformation was noted where the left bronchus ended blindly with a sac.

Overall, only $4.5 \%$ of the patients experienced complications during or after the procedure (Figure 3 ). Desaturation was the most common type of complication. 
Figure 3. Peri-procedural complications of FFB-LMA. A. number of patients with and without peri-procedural complications. B. Distribution of types of complications observed. Desaturation was classified as minor desaturation (a drop of $\mathrm{SpO} 2$ up to 90\%) and major desaturation (a drop of the $\mathrm{SpO}_{2}$ below 90\%).
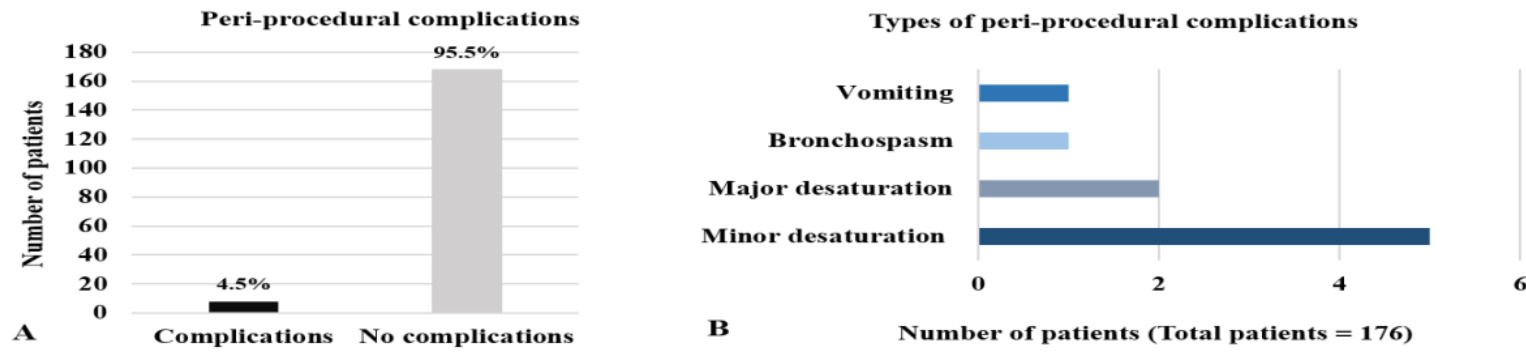

Logistic regression analysis was carried out to assess the risk factors for the development of periprocedural complications. Initially, univariate analysis showed that only the longer duration of the procedure had a significant effect. Subsequently, multivariate analysis was conducted considering all the variables considered in univariate analysis, which also showed that the longer duration of the procedure increased the risk of perioperative complications by nearly 15 times (Table 2 ).

\section{Discussion}

In an era of increased risk of transmission of respiratory infections such as Covid-19 from patients to health care personnel, increased use of FFB, compared to rigid bronchoscopy, is vital and safe as the operator is not directly exposed to the patient's airway. ${ }^{9}$ The current study showed that the FFB-LMA has a good efficacy and a lower complication profile encouraging its widespread use. Similar to several other studies, ${ }^{6,10,11}$ the commonest indication for FFB in the current study was related to respiratory tract infections, mainly pneumonia. On the contrary, in the study by Shirzadi et al., the most common indication was the extraction of foreign bodies. ${ }^{12}$ However, we observed an age category-based variation in the indications for FFB. However, none of the comparable studies had evaluated the indications based on age groups.

Key determinants of the success of FFB-LMA are its efficacy and the diagnostic yield. Overall, clinically significant abnormalities were detected in $87 \%$ of the patients in the current study. The overall diagnostic yield of FFB varied between studies $\left(54 \%^{10}, 68.3 \%{ }^{12}, 72 \%^{6}, 83.6 \%^{7}\right)$. However, the method of airway maintenance and the patient inclusion criteria varied between these studies. Among the patients with suspected foreign body aspirations $(n=39)$, foreign bodies were identified and extracted only in $17(43.6 \%)$ patients $(100 \%$ success). However, airway abnormalities were noted in $27(69.2 \%)$ cases, and some needed suction of thick secretions $(\mathrm{n}=7,18 \%)$ and BAL $(n=5,12.8 \%)$ due to ongoing inflammation. Even though FFB is endorsed as the procedure of choice for the diagnosis of foreign body aspirations, the use of rigid bronchoscopy is considered as the gold standard for their extraction ${ }^{13}$. However, a study by Madkour et al. showed that FFB through LMA under general anaesthesia is a safe and effective method of foreign body retrieval in paediatric patients. $^{7}$

Naguib et al. showed that complications of FFB were lower when done with LMA, compared to other methods of airway maintenance. ${ }^{14}$ Overall, complications were low in our patient cohort at $4.5 \%$. However, the classification of the complications varies between studies making comparisons difficult. Based on the method of classification of complications in the study by Blic et al., in the current study, $1.7 \%$ had major complications, and $2.84 \%$ had minor complications. ${ }^{8}$ Similarly, in the study by Keskin et al., major and minor complications were $2.4 \%$ and $5.6 \%$ respectively. Multivariate analysis was conducted to see the effect of certain variables and risk factors for the development of peri-procedural 
complications. Only the duration of the procedure was found to impose a risk of developing complications (OR-15.5). It was observed that all the FFB-LMA procedures that took longer time durations to complete had been performed by the trainees. The procedures done by consultant ENT surgeon took mostly 10-20 minutes. With further training and experience of these trainees, the procedural time and thus the risk of complications of FFB-LMA could be reduced.

Similarly, a comparable study by Keskin et al. showed that only the duration of the procedure but not the age, weight, gender, ASA category, or the dimension of the LMA were imposing a risk of developing complications during FFB performed through LMA. ${ }^{6}$

As the data were collected retrospectively from anaesthetics charts and BHTs, it is possible that certain periprocedural complications, especially minor complications were not recorded. However, most of the time, all significant anaesthetic complications are recorded on anaesthetics charts, and surgical complications are recorded on BHT. Therefore, the possibility of missing any significant peri-procedural complications is minute. Furthermore, potential errors in recording the procedural duration could have affected the analysis as the data were recorded retrospectively.

\section{Conclusion}

FFB-LMA is a safe and effective procedure, with minimum peri-procedural complications, based on the studied paediatric patient sample in Sri Lanka.

\section{References}

1. Midulla F, de Blic J, Barbato A, Bush A, Eber E, et al. Flexible endoscopy of paediatric airways. Eur Respir J 2003; 22:698-708 https://doi:10.1183/09031936.02.00113202

2. Williams A, George C, Atul PS, Sam S, Shukla S. An audit of morbidity and mortality associated with foreign body aspiration in children from a tertiary level hospital in Northern India. African J Paediatr Surg 2014; 11:287-92

https://doi.org/10.4103/0189-6725.143129

PMid:25323175
3. Roberts S, Thornington RE. Paediatric bronchoscopy. Contin Educ Anaesthesia,Crit Care Pain 2005; 5:41-4. https://doi.org/10.1093/bjaceaccp/mki015

4. Stahl D, Richard K, Papadimos T. Complications of bronchoscopy: A concise synopsis. Int J Crit Illn Inj Sci 2015; 5:189. https://doi.org/10.4103/2229-5151.164995 PMID: 26557489 PMCID: PMC4613418

5. Fadaizadeh L, Hoseini MS, Bagheri M. Anaesthesia Management During Interventional Bronchoscopic Procedures: Laryngeal Mask Airway or Rigid Bronchoscope. Turkish J Anesth Reanim 2014; $\quad$ 42(6): 302-307 https://doi.org/10.5152/TJAR.2014.85579 PMID: 27366442 PMCID: PMC4894128

6. Keskin G, Akin M, Senayli Y, Saydam S, Kurt DT, et al. Laryngeal mask airway application during flexible fiberoptic bronchoscopy in pediatric patients: Evaluation of 125 cases. Anestezi Derg 2019; 27:24-9. https://doi.org/10.5222/jarss.2019.36025

7. Madkour A, Sharkawy S, Elmansoury A. The efficacy of fiberoptic bronchoscopy through laryngeal mask airway in pediatric foreign body extraction. Egypt J Bronchol 2014; 8:57. https://doi.org/10.4103/1687-8426.137354

8. de Blic J, Marchac V, Scheinmann P. Complications of flexible bronchoscopy in children: Prospective study of 1,328 procedures. Eur Respir J 2002; 20:1271-6. https://doi.org/10.1183/09031936.02.0207200 1

PMID: 12449184

9. Francom CR, Javia LR, Wolter NE, Lee GS, Wine $\mathrm{T}$, et al. Pediatric laryngoscopy and bronchoscopy during the COVID-19 pandemic: A four-center collaborative protocol to improve safety with perioperative management strategies and creation of a surgical tent with disposable drapes. Int $J$ Pediatr Otorhinolaryngol 2020;134. https://doi.org/10.1016/j.ijporl.2020.110059 PMID: 32339971 PMCID: PMC7172675

10. Kabra SK, Lodha R, Ramesh P, Sarthi M. Fiberoptic bronchoscopy in children: An audit 
Dissanayake et al. Sri Lankan Journal of Anaesthesiology: 29(2): 88-94 (2021)

from a tertiary care center. Indian Pediatr 2008; 45(11):917-9.

11. Kirvassilis F, Gidaris D, Ventouri M, Zampouri A, Mylona M, et al. Flexible fiberoptic bronchoscopy in Greek children. Hippokratia 2011; 15(4):312-5.

12. Shirzadi R, Navaei S, Razavi - Khorasani N, Masiha F, Mirlohi SH, et al. Indications and complications of flexible fiberoptic bronchoscopy in children: A 5-year experience at a tertiary care hospital in Iran. Iran J Pediatr 2020; $30 \quad 30 \quad$ (2): $\quad$ e92535. https://doi.org/10.5812/ijp.92535
13. Korlacki W, Korecka K, Dzielicki J. Foreign body aspiration in children: Diagnostic and therapeutic role of bronchoscopy. Pediatr Surg Int 2011; 27(8):833-7

https://doi.org/10.1007/s00383-011-2874-8

PMID: 21400033

PMCID: PMC3136687

14. Naguib ML, Streetman DS, Clifton S, Nasr SZ. Use of laryngeal mask airway in flexible bronchoscopy in infants and children. Pediatr Pulmonol 2005; 39(1):56-63.

https://doi.org/10.1002/ppul.20139

PMID: 15558607 